\title{
ORIGINAL ARTICLE Neuroplasticity in response to cognitive behavior therapy for social anxiety disorder
}

\author{
KNT Månsson ${ }^{1,2}$, A Salami ${ }^{3,4}$, A Frick ${ }^{5}$, P Carlbring ${ }^{6}$, G Andersson ${ }^{1,7}$, T Furmark $^{5}$ and C-J Boraxbekk A $^{4,8}$
}

Patients with anxiety disorders exhibit excessive neural reactivity in the amygdala, which can be normalized by effective treatment like cognitive behavior therapy (CBT). Mechanisms underlying the brain's adaptation to anxiolytic treatments are likely related both to structural plasticity and functional response alterations, but multimodal neuroimaging studies addressing structure-function interactions are currently missing. Here, we examined treatment-related changes in brain structure (gray matter (GM) volume) and function (blood-oxygen level dependent, BOLD response to self-referential criticism) in 26 participants with social anxiety disorder randomly assigned either to CBT or an attention bias modification control treatment. Also, 26 matched healthy controls were included. Significant time $\times$ treatment interactions were found in the amygdala with decreases both in GM volume (family-wise error (FWE) corrected $\left.P^{\mathrm{FWE}}=0.02\right)$ and BOLD responsivity $\left(P^{\mathrm{FWE}}=0.01\right)$ after successful CBT. Before treatment, amygdala GM volume correlated positively with anticipatory speech anxiety $\left(P^{\mathrm{FWE}}=0.04\right)$, and CBT-induced reduction of amygdala GM volume (pre-post) correlated positively with reduced anticipatory anxiety after treatment ( $\left.P^{\mathrm{FWE}} \leqslant 0.05\right)$. In addition, we observed greater amygdala neural responsivity to self-referential criticism in socially anxious participants, as compared with controls $\left(P^{\mathrm{FWE}}=0.029\right)$, before but not after CBT. Further analysis indicated that diminished amygdala GM volume mediated the relationship between decreased neural responsivity and reduced social anxiety after treatment $(P=0.007)$. Thus, our results suggest that improvement-related structural plasticity impacts neural responsiveness within the amygdala, which could be essential for achieving anxiety reduction with CBT.

Translational Psychiatry (2016) 6, e727; doi:10.1038/tp.2015.218; published online 2 February 2016

\section{INTRODUCTION}

The brain is remarkably adaptive. Neuroimaging studies in animals and humans have shown multiple facets of plasticity, ${ }^{1,2}$ manifested as structural changes induced by environment, ${ }^{3}$ learning, ${ }^{4}$ behavior $^{1,5}$ and emotions. ${ }^{6}$ Similar to structural plasticity, emotions alter neural responsivity. 7,8 Although the notion that the human brain is adaptive has extensive support from unimodal brain imaging studies, integrative models of the brain's functional changes in relation to structural plasticity are largely lacking, and multimodal studies are needed to improve our understanding of the adaptive brain.9,10 For instance, using a longitudinal multimodal-imaging approach, Nyberg and colleagues $^{11,12}$ demonstrated that age-related reductions in prefrontal functional response to a cognitive task are driven by local gray matter (GM) atrophy. The observation that neural response alterations over time are accounted for by structural changes may have implications for research in psychiatric disorders. For example, a recent large study found that both GM volume and neural responsivity in the amygdala were associated with symptoms of separation anxiety, ${ }^{13}$ suggesting dependence between anxietyrelated brain structure and function.

The amygdala, anterior cingulate cortex (ACC), insula and hippocampus, have central roles in the acquisition and expression of fear. ${ }^{14}$ Exaggerated neural response in these regions has commonly been reported in anxiety disorders. ${ }^{15-17}$ In comparison to functional activation studies, volumetric differences between anxiety patients and healthy controls have been investigated less frequently, ${ }^{18-24}$ and inconsistent findings are present, for example, in volumetric studies on social anxiety. ${ }^{25}$ Examinations of both structure and function concomitantly in anxious patients are largely lacking, ${ }^{26}$ and it has not been studied whether anxietyrelated structure and neural activity are simultaneously changed by effective treatments. Treatments targeting anxiety, like cognitive behavior therapy $(\mathrm{CBT})^{27-31}$ and psychotropic medication (for example, with selective serotonin reuptake inhibitors), ${ }^{28,32}$ have been shown to decrease neural responsivity in the amygdala. In line with this, Mahan and Ressler ${ }^{33}$ suggested that synaptic plasticity in the amygdala may be an important target for treatment of posttraumatic stress disorder. While there is limited literature on structural changes with anxiety-reducing pharmacologic agents ${ }^{34,35}$ or psychotherapy, ${ }^{36}$ the results are mixed and the conclusions are restricted by not including a treatment control group.

The present randomized controlled trial (RCT) examined CBTrelated changes in the brain using a multimodal neuroimaging approach. Hence, we evaluated the relationship between structural neuroplasticity (GM volume) and functional changes (bloodoxygen level dependent, BOLD responsivity to self-referential

\footnotetext{
${ }^{1}$ Division of Psychology, Department of Behavioural Sciences and Learning, Linköping University, Linköping, Sweden; ${ }^{2}$ Department of Adult Psychiatry, PRIMA Barn och Vuxenpsykiatri, Stockholm, Sweden; ${ }^{3}$ Department of Neurobiology, Care Sciences and Society, Aging Research Center, Karolinska Institutet, Stockholm, Sweden; ${ }^{4}$ Umeå Center for Functional Brain Imaging, Umeå University, Umeå, Sweden; ${ }^{5}$ Department of Psychology, Uppsala University, Uppsala, Sweden; ${ }^{6}$ Department of Psychology, Stockholm University, Stockholm, Sweden; ${ }^{7}$ Department of Clinical Neuroscience, Psychiatry Section, Karolinska Institutet, Stockholm, Sweden and ${ }^{8}$ CEDAR, Center for Demographic and Aging

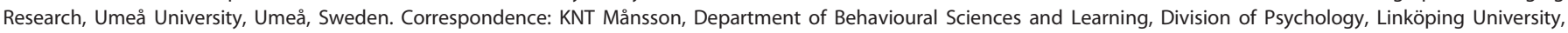
Linköping SE-581 83, Sweden.
}

E-mail: kristoffer.nt.mansson@liu.se

Received 21 July 2015; revised 15 October 2015; accepted 2 December 2015 
criticism) $^{37}$ in participants with social anxiety disorder (SAD) who were treated with CBT or an attention-training control treatment. ${ }^{27}$ Brain parameters were also related to a matched healthy control group to evaluate pre-treatment differences and normalization effects of treatment. We expected concomitant treatment-related changes in GM volume and functional response in the brain's fear circuitry (that is, the amygdala, ACC, insula and hippocampus). ${ }^{17}$ Mediation analysis was conducted to determine the path for reduced social anxiety after treatment, that is, whether structural neuroplasticity or altered neural response mediated the relationship between the other and symptom improvement with CBT.

\section{MATERIALS AND METHODS}

\section{Participants}

Fifty-two right-handed participants were included (see Table 1), 26 with a primary diagnosis of SAD according to the structured clinical interview for DSM-IV axis I (SCID-1) $)^{38}$ and 26 healthy controls matched on age, sex and educational level, and free from psychiatric disorders as assessed by the Mini-International Neuropsychiatric Interview (MINI). ${ }^{39}$ Prior to the diagnostic telephone interview, participants answered Internetadministered self-report questionnaires regarding social anxiety (for example, Liebowitz Social Anxiety Scale - Self-report version (LSAS-SR) ${ }^{40}$ see online Supplementary Material), depression (for example, Montgomery-Åsberg Depression Rating Scale-Self-report version (MADRS-S) ${ }^{41}$ and magnetic resonance safety. Eight SAD participants were on prescription medication throughout the study, but the selective serotonin reuptake inhibitors dose had been stable for at least 3 months prior to treatment initiation. As shown in Table 1, SAD participants had higher scores on social anxiety and depressive symptoms than the healthy controls.

The study was registered at ClinicalTrials.gov (id: NCT01312571), and approval was obtained from the regional ethics committee. All participants gave written informed consent prior to participation.

\section{Procedure and design}

The present paper is part of a RCT previously described in detail. ${ }^{27,42}$ In short, participants with SAD were treated with Internet-delivered CBT or Internet-delivered Attention Bias Modification (ABM), and an independent researcher executed the randomization. Magnetic resonance imaging (MRI) assessments were performed before as well as 9 weeks after treatment (pre-post). The matched healthy controls underwent only one MRI assessment. The SAD participants were not offered economic compensation, but the healthy controls received about 125 USD and a cinema ticket.

\section{Clinical assessment}

An independent clinical psychologist, blind to the experimental conditions, determined clinical response rates using the Clinical Global ImpressionImprovement scale (CGI-l; scores 1 or 2 , that is, much or very much improved defining treatment responders). ${ }^{43}$ Moreover, social anxiety selfreport questionnaires were administrated at pre- and post-treatment (for example, LSAS-SR). ${ }^{40}$ We were also interested in state-related social anxiety, ${ }^{44}$ so after the MRI assessment the participants performed a 2-min public speaking task. Both after the initial anatomical image acquisition, and prior to the speech, the participants rated subjective units of discomfort. Fear and distress were rated separately on a scale from 0 to 100 (min-max), and anxiety was calculated as the mean of these measures.

\section{Treatment}

The CBT in the present study was delivered over a period of 9 weeks, and the therapist provided written feedback once a week. Several independent RCTs on guided Internet-delivered CBT for SAD show robust effects, ${ }^{45,46}$ and comparable improvement to conventional CBT delivered face-toface. ${ }^{47}$ We have previously demonstrated that the present treatment, compared with $A B M$, reduced anxiety-related amygdala responsivity to emotional faces. ${ }^{27}$

$A B M$ is a computer-assisted intervention aimed at improving a threatdetecting cognitive bias that characterizes SAD. ${ }^{48}$ In concordance with previous studies, the ABM was delivered twice a week over 4 weeks. ABM has been described in detail elsewhere. ${ }^{27,42,49}$ While ABM has shown promise, ${ }^{50,51}$ it has been found to be less effective when delivered through the Internet ${ }^{49}$ and we used ABM as a control in the present trial. See online Supplementary Material for further details.

\section{BOLD-fMRI experimental task}

BOLD response to self-referential criticism was recorded using functional magnetic resonance imaging (fMRI) and an information-processing paradigm developed by Blair et al. ${ }^{37}$ Essentially, the participants were instructed to read sentences and press a button using their right hand as a confirmation. The sentences contained criticism targeting the participant or others. Functional responses to self and other referential information were recorded for a maximum of $2500 \mathrm{~ms}$, and fixation crosses (' + '; $2500 \mathrm{~ms}$ ) were randomly interspersed between the sentences. In addition, each sentence and fixation cross was separated by a cross or circle presented for $500 \mathrm{~ms}$ (see also Supplementary Figure S3). Stimuli were demonstrated using the E-prime 2.0 software (Psychology Software Tools, Pittsburgh, PA, USA), projected on a screen and viewed through a tilted mirror attached to the head coil. For more details, see the study Månsson et $a .^{42}$

\section{Image acquisition and preprocessing}

Neuroimaging was performed in a 3 T scanner (General Electric, Madison, WI, USA), equipped with a 32-channel head coil. Structural T1-weighted images were acquired with voxel size: $0.5 \times 0.5 \times 1 \mathrm{~mm}^{3}$ ( 180 slices; field of view: $250 \mathrm{~mm}$ ). For functional images, the following parameters were used: echo time: $30 \mathrm{~ms}$, repetition time: $2000 \mathrm{~ms}$, flip-angle: $80^{\circ}$, field of view: $250 \times 250 \mathrm{~mm}^{2}$, matrix size: $96 \times 96$, in-plane resolution: $2.6 \times 2.6 \mathrm{~mm}$. Thirty-seven slices with a thickness of $3.4 \mathrm{~mm}$ were acquired every 2000 ms. Ten dummy scans were run before the image acquisition started to avoid signals resulting from progressive saturation.

The Statistical Parametric Mapping Software v. 8 (SPM8; Wellcome Department of Cognitive Neurology, London, UK) and the MATLAB (Mathworks, Natick, MA, USA) were used to perform neuroimaging

Table 1. Demographic and clinical characteristics of SAD participants and healthy controls

\begin{tabular}{|c|c|c|c|}
\hline Age, years, mean (s.d.) & $32.27(9.7)$ & $32.23(10.5)$ & $t(51)=0.01, P=0.989$ \\
\hline Range, years & $19-57$ & $18-57$ & - \\
\hline Highest educational level, n (\%) & & & $X^{2}=0.42, P=0.810$ \\
\hline Completed university & $9(35)$ & $11(42)$ & \\
\hline Current university & $10(38)$ & $8(31)$ & \\
\hline LSAS-SR, mean (s.d.) & $76.31(18.7)$ & $20.53(11.4)$ & $t(51)=12.97, P<0.001$ \\
\hline MADRS-S, mean (s.d.) & $15.73(6.6)$ & $6.27(4.9)$ & $t(51)=5.87, P<0.001$ \\
\hline Anticipatory speech anxiety, mean (s.d.) & $50.15(24.1)$ & $13.44(16.0)$ & $t(51)=6.48, P<0.001$ \\
\hline
\end{tabular}


analyses. The T1-weighted images were preprocessed using the VBM8 toolbox (http://dbm.neuro.uni-jena.de/vbm/download). The Voxel-Based Morphometry v. 8 (VBM8) toolbox calculates modulated normalized GM volumes and allows for comparing tissue amounts while controlling for individual brain sizes (default settings were used). To identify outliers, quality control was carried out using the sample homogeneity test, and we found that covariance was within 2 s.d. Thus, no outliers were excluded. VBM8 preprocessing was performed in 3 steps; (a) longitudinal MRI data assessment on SAD participants (pre- vs post-treatment; $n=23 \times 2$; that is, three participants withdrew from the post-MRI assessment), (b) casecontrol differences at baseline (SAD vs healthy control; $n=26+26$ ) and (c) case-control differences following CBT $(n=11+26)$. Structural scans were segmented into gray and white matter, and the GM volumes were nonlinearly normalized to the Montreal Neurological Institute (MNI) template, modulated and smoothed using an $8 \mathrm{~mm}$ full-width half-maximum isotropic Gaussian kernel.

Functional MRI data were first rigidly aligned to the middle image volume of each run to correct for head movements. The realigned images were then corrected for acquisition time differences between slices within each volume. A within-subject rigid registration was conducted to align functional and structural images together. For the 23 SAD participants who underwent the post-treatment MRI, we co-registered the functional scans to a longitudinal mean structural image (if post-treatment images were missing, the pre-treatment image was used). For healthy controls, the functional scans were co-registered to the structural image. Functional scans were subsequently warped to MNI152 standard space (using the transformation parameters that normalized GM images into the MNI space) and smoothed with an $8 \mathrm{~mm}$ full-width half-maximum isotropic Gaussian kernel. Thus, both $\mathrm{FMRI}$ and VBM images were in the same space and had the same voxel size (that is, $1.5 \times 1.5 \times 1.5 \mathrm{~mm}^{3}$ ).

In the BOLD-fMRI paradigm, subject-specific contrasts (self-referential criticism vs other referential criticism) were generated with voxel-wise general linear models. Each condition was modeled as a box-car function, convolved with the hemodynamic response function and filtered using a $128 \mathrm{~s}$ high-pass filter. In addition, six motion parameters derived from the realignment algorithm were included in the model to account for motion artifacts.

\section{Data analysis}

Demographics, clinical data and mediation analyses were evaluated using the STATA Statistical Software, v. 13.1 (STATA, College Station, TX, USA) and SPSS Statistics, v. 19.0 (IBM, Armonk, NY, USA).

Regions of interest (ROI) in all neuroimaging analyses were the left and right amygdala, ACC, insula and hippocampus, that is, regions of the fear circuitry ${ }^{17}$ affected in SAD. ${ }^{15,16,25,52}$ ROls were defined using the AAL Atlas from the Wake Forest University PickAtlas, ${ }^{53}$ and the significance threshold level was set at $P<0.05$ with family-wise error (FWE) correction on voxelwise comparisons within each ROI. Whole-brain analyses used $P<0.001$ as the significance threshold and 10 voxels as the extent threshold.

The clinical outcome of CBT, relative to the ABM, on the CGI-I was calculated using a $X^{2}$-test, and self-reported effects were evaluated using repeated measure MANOVA (multivariate analysis of variance on social anxiety questionnaires) or MANCOVA (multivariate analysis of covariance on state-related anxiety).

Time (pre- and post-treatment) $\times$ treatment $(C B T$ and $A B M)$ interactions were assessed using the flexible factorial design. Significant interactions were further qualified with pairwise comparisons, that is, paired $t$-tests within the CBT group. Each imaging modality was analyzed separately. The association between social anxiety (that is, LSAS-SR and anticipatory speech anxiety) and either GM volume or BOLD responsivity was calculated in separate regression models, both at pre-treatment and as a function of CBT. Change in GM volume $(\triangle G M)$, and BOLD response $(\triangle B O L D)$ were calculated using the SPM8 ImCalc function. To assess casecontrol differences in structure and function, as well as putative normalization effects of CBT, we compared GM volume and BOLD responsivity (at pre- and post-treatment) in SAD participants relative to healthy controls using independent two-sample $t$-tests within each image modality.

To explore the most probable path for reduced social anxiety after treatment, that is, structural neuroplasticity or change on neural response, we performed mediation analyses in accordance with the Shrout and Bolger model, ${ }^{54}$ in which sufficient statistical power may be less critical to detect mediation. The outcome measure used in these analyses was social anxiety symptom improvement, that is, changes either in LSAS-SR scores or anticipatory speech anxiety (using separate models). First, we extracted changes in GM volume and BOLD response from voxels exhibiting significant positive correlations $P<0.05$ (FWE corrected at cluster-level) between these measures (the $a$-path). Correlations were established using the Biological Parametric Mapping (BPM) toolbox. ${ }^{55}$ BPM is a multimodalimaging approach that can model change in one modality (for example, BOLD-fMRI) as a function of change in another modality (for example, GM volume) using the general linear model framework. The $b$-path refers to the relationship between the mediator and the outcome (that is, social anxiety improvement). We calculated mediation paths with either change in GM, or change in BOLD response as mediators. The interaction of $a$ - and $b$-path represents the indirect effect, and the predictor-outcome association is referred to as the direct effect (the $c^{\prime}$-path). The interaction term was estimated using bootstrap resampling $(n=5000)$ to jointly estimate the direct and indirect effects, therefore minimizing the dependence on normally distributed data. ${ }^{54}$

\section{RESULTS}

Treatment outcome and compliance

As previously reported, ${ }^{27}$ the clinician administrated CGI-I assessments revealed significantly more participants responding positively to the CBT $(61 \%, 8 / 13)$ than to the $A B M$ control treatment $\left(23 \%, 3 / 13 ; X^{2}=3.94, P=0.047\right)$, and on the self-report questionnaires, we found similar results in favor of CBT. ${ }^{27}$ Furthermore, when controlling for pre-treatment level, anticipatory speech anxiety decreased more with CBT than with ABM (time $\times$ treatment; Wilks's $\left.\lambda=0.678, F_{2,21}=4.98, P=0.017\right)$, see Supplementary Table S1, and Supplementary Figure S4.

On average, the CBT participants completed eight (out of nine) modules of treatment. In addition to completing the modulebased assignments, the participants were required to earn at least 95\% correct answers on a multiple-choice quiz about CBT every week. The ABM control participants earned an average of $98.4 \%$ (16 383/16 640) correct responses on the attentional shifting task, and they completed all training sessions.

Treatment effects on brain structure and neural responsivity

Time $\times$ treatment interactions indicated that left amygdala GM volume and right amygdala BOLD response decreased significantly more with CBT compared with the ABM control treatment (see Table 2 and Figure 1). Similarly, follow-up pairwise comparisons within the CBT group suggested decreased left amygdala GM volume and right amygdala BOLD responsivity after treatment (see Table 2).

Table 2. Structural and functional response alterations in participants treated with effective $C B T$, in comparison to the $A B M$ control treatment (time $\times$ treatment interactions)

\begin{tabular}{|c|c|c|c|c|c|c|c|}
\hline \multirow[t]{2}{*}{$\begin{array}{l}\text { Analyses and brain } \\
\text { regions }\end{array}$} & \multirow[t]{2}{*}{$M R I$} & \multicolumn{3}{|c|}{$\begin{array}{c}\text { MNI } \\
\text { coordinates }\end{array}$} & \multirow{2}{*}{$\begin{array}{l}\text { Maximum } \\
\text { Z value }\end{array}$} & \multirow[t]{2}{*}{ Voxels } & \multirow[t]{2}{*}{$\mathrm{P}^{F W E}$} \\
\hline & & $\mathrm{x}$ & $\mathrm{y}$ & z & & & \\
\hline \multicolumn{8}{|c|}{$2 \times 2$ interactions (pre-treatment vs post-treatment $\times C B T$ vs $A B M$ ) } \\
\hline L Amygdala & GM & -20 & -1 & -21 & 3.30 & 69 & 0.024 \\
\hline R Amygdala & GM & 22 & 2 & -21 & 2.70 & 27 & 0.122 \\
\hline L Amygdala & BOLD & -26 & -7 & -17 & 2.05 & 26 & 0.218 \\
\hline R Amygdala & BOLD & 28 & 0 & -16 & 3.28 & 140 & 0.015 \\
\hline \multicolumn{8}{|c|}{ Main effects of CBT (pre-treatment $>$ post-treatment) } \\
\hline L Amygdala & GM & -20 & -1 & -21 & 3.12 & 61 & 0.060 \\
\hline R Amygdala & GM & 22 & 2 & -21 & 2.15 & 17 & 0.395 \\
\hline L Amygdala & BOLD & - & - & - & $<0.0$ & & \\
\hline R Amygdala & BOLD & 28 & 2 & -16 & 2.89 & 78 & 0.061 \\
\hline
\end{tabular}



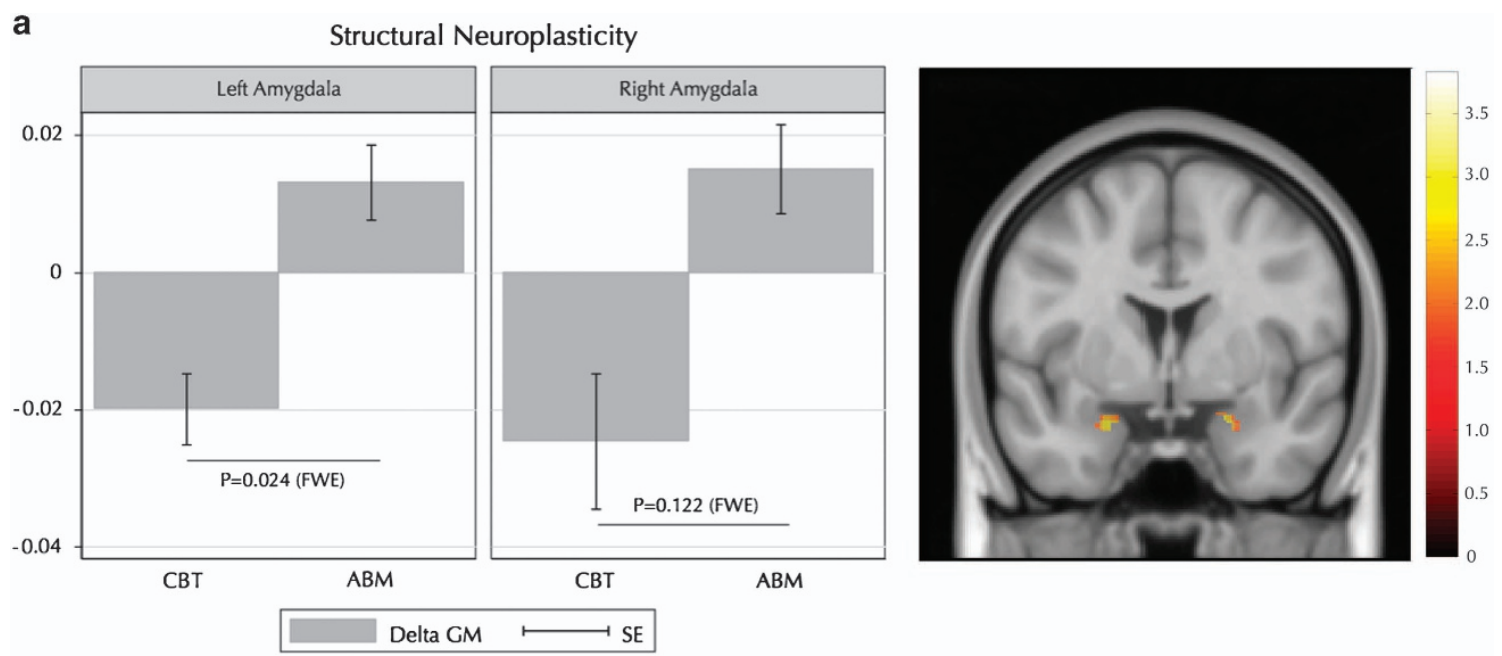

b

Functional Changes
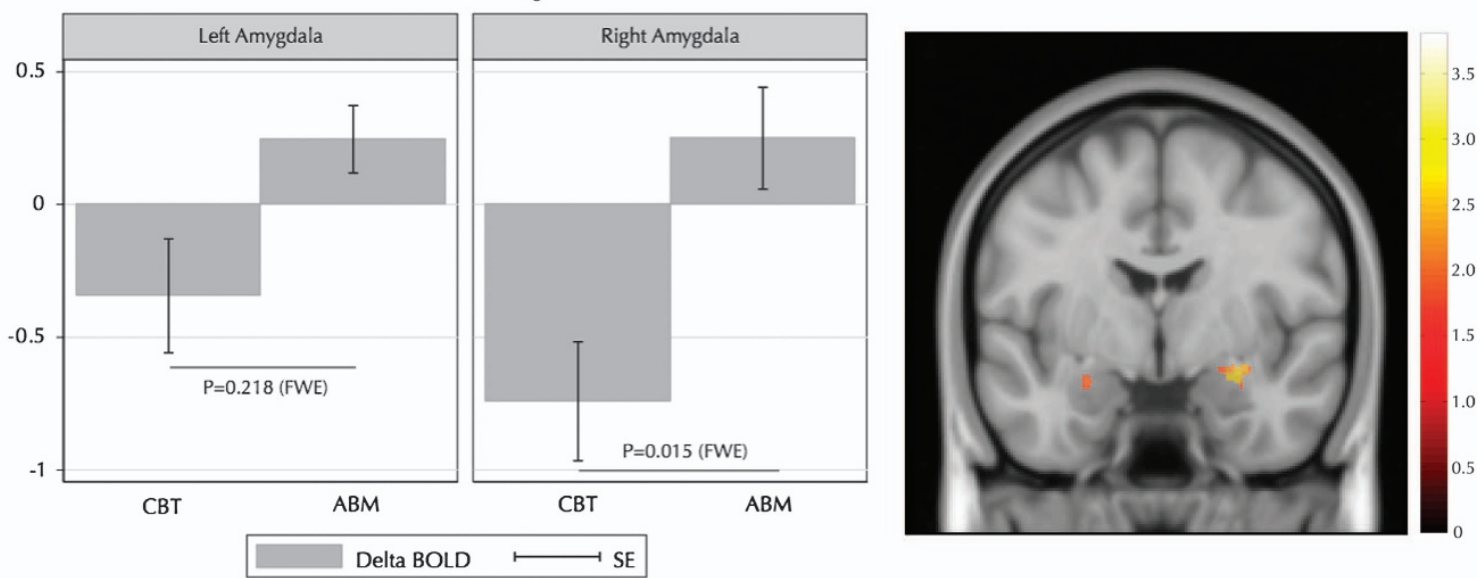

Figure 1. Changes in (a) amygdala GM volume and (b) amygdala BOLD response to self-referential criticism, sorted by treatment and hemisphere. The $y$-axis shows change in beta-weight values, and lower beta-weights correspond to reduced GM volume and BOLD responsivity over time (pre-post). Error bars represent s.e. ABM, attention bias modification; CBT, cognitive behavior therapy; FWE, family-wise error corrected $P$-value; GM, gray matter volume.

Whole-brain analysis of structural change showed that the GM volumes of the dorsomedial prefrontal cortex (Brodmann area 8) and the bilateral precuneus were more greatly reduced after CBT than ABM (see Supplementary Table S2). Whole-brain BOLD-signal analyses only revealed a greater reduction in the activation of the right amygdala (xyz: 29,1,-16; $Z=3.28, P<0.001, k=15$ voxels) in CBT compared with ABM participants.

Relationship between social anxiety symptomatology, brain structure and neural responsivity

Pre-treatment GM volume in the left amygdala was positively correlated with the level of anticipatory speech anxiety in SAD participants (xyz: $-24,-4,-12 ; Z=2.96, P^{\mathrm{FWE}}=0.04 ; k=273$ voxels, see Figure 2). As reported in Supplementary Table S3, whole-brain analysis additionally showed that GM volume of the left and right precuneus was positively correlated with anticipatory speech anxiety.

CBT-induced reductions of the GM volumes of the bilateral amygdala and the insula were positively associated with decreased levels of anticipatory speech anxiety (left amygdala, $x y z:-16,-3,-18 ; Z=2.83, P^{\mathrm{FWE}}=0.05, k=73$ voxels; right amygdala, $x y z: 22,6,-18 ; Z=3.42, P^{\mathrm{FWE}}=0.01, k=131$ voxels; see Figure 2; left insula, $x y z$ : $-40,14,1 ; Z=4.02, P^{\mathrm{FWE}}=0.02, k=468$ voxels; right insula, xyz: $28,12,-18 ; Z=3.69, P^{\mathrm{FWE}}=0.04, k=128$ voxels). Whole- brain analysis revealed anxiety-related reductions of the GM volume in the left fusiform gyrus (Supplementary Table S4).

Contrary to our expectations, GM volume was not associated with general social anxiety symptomatology as measured with LSAS-SR; nor was BOLD responsivity significantly correlated with pre-treatment severity or pre-post improvement of symptoms (anticipatory speech anxiety or LSAS-SR).

Comparisons of brain structure and neural response between SAD participants and healthy controls

Before treatment there was no significant difference between SAD participants and healthy controls in GM volume within the fear neurocircuitry $\left(Z<2.99\right.$, $\left.P^{\mathrm{FWE}}>0.374\right)$. Amygdala BOLD response to self-referential criticism was elevated in participants with $S A D$ compared with the healthy controls (right amygdala, $x y z: 27,-8,-$ $12 ; Z=3.04, P^{\mathrm{FWE}}=0.029, k=150$ voxels; with a trend in the left amygdala (xyz: $-26,-2,-11 ; Z=2.54, P^{\mathrm{FWE}}=0.088, k=90$ voxels). The post-CBT amygdala response did not differ significantly from that of healthy controls $\left(Z<.50, P^{\mathrm{FWE}}>0.743\right)$, indicating normalization through CBT. Result from the whole-brain analysis is reported in Supplementary Table S5. 
a
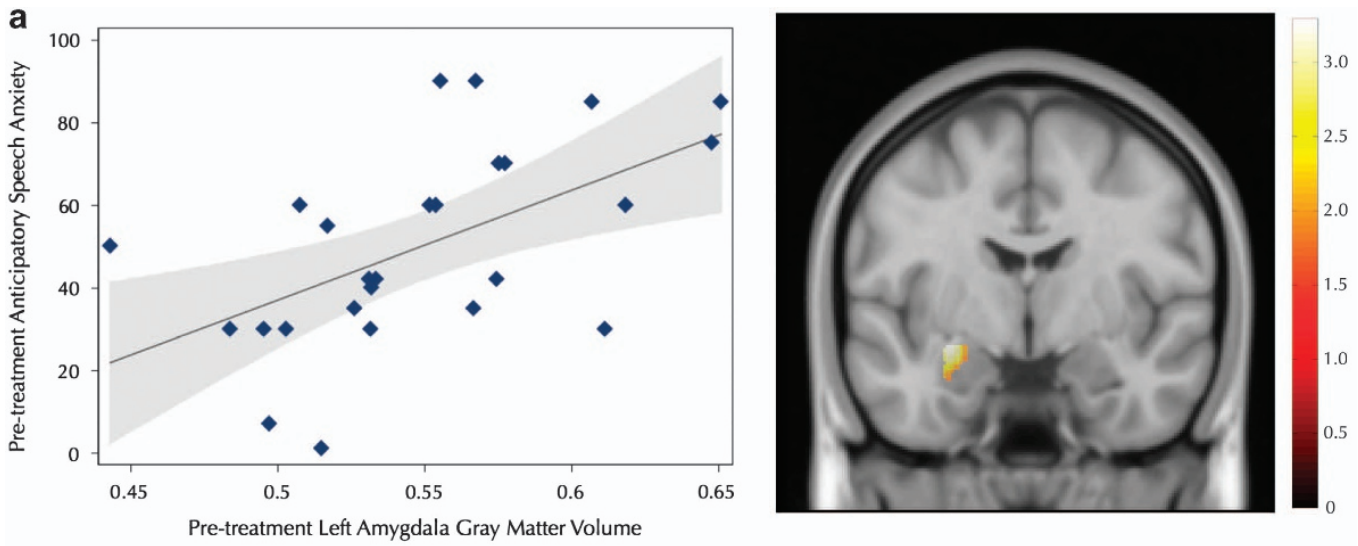

b

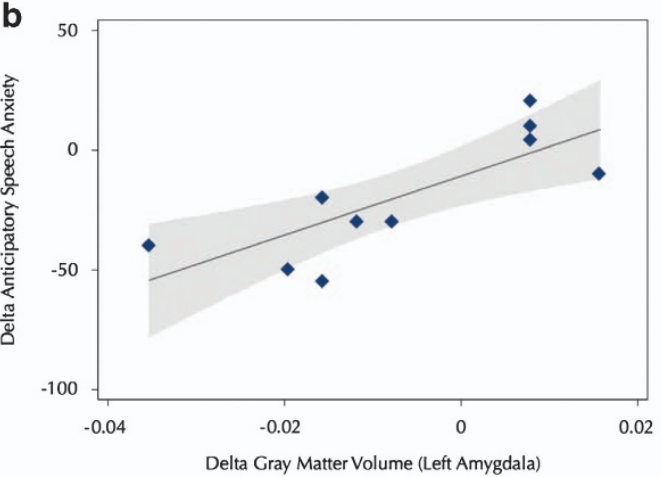

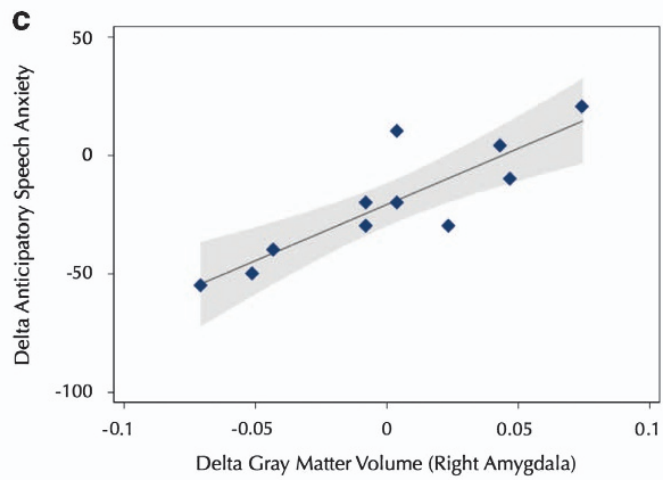

Figure 2. Brain-behavior correlations showing that (a) pre-treatment gray matter volume in the left amygdala was associated with enhanced levels of anticipatory speech anxiety, and (b and $\mathbf{c}$ ) reduced amygdala gray matter volume (left and right respectively) was positively associated with improved symptoms of anticipatory speech anxiety after cognitive behavior therapy. Gray shading corresponds to $95 \%$ confidence intervals.

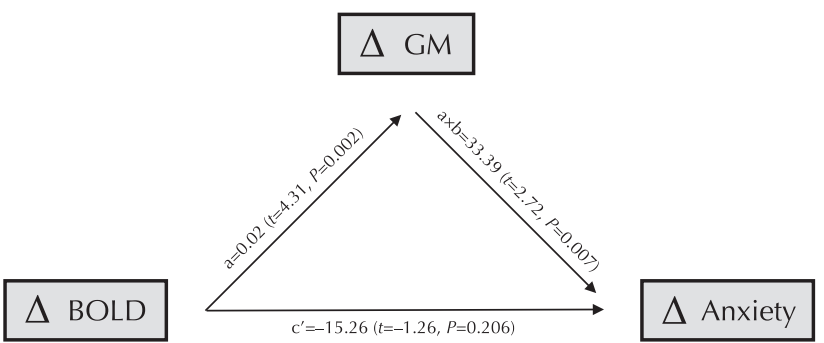

Figure 3. The mediation paths supported reduced gray matter volume as the mediator of the relation between diminished amygdala BOLD responsivity (predictor) and improved anticipatory speech anxiety after cognitive behavior therapy. GM, gray matter.

\section{Mediation analysis}

Mediation analysis was conducted to determine the most probable brain path for improvement in anticipatory speech anxiety. Within the CBT group, the $a$-path was significant in both amygdalae, indicating that structural plasticity was interrelated with diminished amygdala neural responsivity (right amygdala, $x y z$ : $29,1,-22 ; Z=3.14, P^{\mathrm{FWE}}=0.001$ at cluster-level, $k=71$ voxels; left amygdala, $x y z$ : $-21,2,-20 ; Z=2.45, P^{\mathrm{FWE}}=0.029$ at cluster-level; $k=40$ voxels).

In the right amygdala, reduced GM volume mediated the relationship between reduced BOLD response and symptom improvement $(a \times b$-path, indirect effect: $\beta=33.39,95 \% \mathrm{Cl}=9.32$ to $57.45, P=0.007)$. The direct effect was not significant ( $c^{\prime}$-path: $\beta=-15.26,95 \% \mathrm{Cl}=-38.93$ to $8.40, P=0.206$; see also Figure 3 ). Furthermore, without controlling for the indirect effect, the $c$-path suggested an opposite relationship $(\beta=18.12, P=0.104)$ between the predictor and the outcome, that is, an inverse association. The other probable path, that is, BOLD response as mediator, was not significant $(\beta=-479.90,95 \% \mathrm{Cl}=-1469.93$ to $510.14, P=0.342)$.

\section{Supplementary analyses}

As detailed in the online Supplementary Materials, we found no pre-treatment differences in clinical and demographic (for example, age and sex) variables, nor did the amygdala characteristics (that is, GM volume or BOLD responsivity) differ between participants allocated to CBT or ABM. Furthermore, we found no clinical, or demographic differences between included participants and the three participants who withdrew from the posttreatment MRI assessment. Finally, we performed an alternative mediation analysis using the CGI-I responder status as the outcome measure, noting a trend-level indirect effect ( $a \times b$-path).

\section{DISCUSSION}

Using a multimodal neuroimaging RCT design, we demonstrate interrelated structural plasticity and altered neural responsivity, within the amygdala, after CBT for social anxiety. Both GM volume and neural responsivity in the bilateral amygdala diminished after effective treatment. Left amygdala GM volume was positively associated with symptom severity before treatment, and amygdala volume decreased significantly with CBT, correlating positively with symptom improvement in both hemispheres. Concomitantly, amygdala hyperresponsivity to self-referential criticism was normalized with CBT, and the mediation paths suggested that reduced amygdala volume mediated the relationship between decreased right amygdala neural response and decreased social anxiety after treatment. 
Thus, we demonstrate that the relationship between CBTinduced attenuation of amygdala hyperresponsivity and social anxiety symptoms is mediated by decreased local GM volume. Similar to our finding on the structure-function relationship in the amygdala, a previous longitudinal study also showed dependence between GM atrophy and age-related cognitive neural responsivity in the prefrontal cortex, ${ }^{11}$ suggesting that the adaptive brain may be best understood in a multimodal context. Hence, we argue that analyses of structural neuroplasticity and concomitant functional changes provide better understanding of how the brain adapts to anxiolytic treatments, which could not be fully explained by each modality separately. Furthermore, our results reinforce the notion that structural neuroplasticity in the amygdala is an important target for psychosocial treatments of anxiety, as previously suggested for pharmacological treatments of posttraumatic stress disorder. ${ }^{33}$

In our whole-brain analyses we found volumetric reduction, but not decreased neural response, following CBT in regions that have been linked to self-referential processing and commonly also in studies of the default mode network (that is, the dorsomedial prefrontal cortex and precuneus), ${ }^{56}$ see Supplementary Tables S2-S4. Resting-state fMRI and neural response in the default mode network have also been suggested to be biomarkers for SAD. ${ }^{57}$ Further, as suggested in a previous BOLD-fMRI study on self-referential criticism, ${ }^{37}$ SAD participants were hyperresponsive in the amygdala. The present results further indicate that the excessive amygdala responsivity is normalized with successful CBT. ${ }^{27-29,58}$ This is consistent with studies demonstrating reduced amygdala responsivity after anxiolytic treatments, ${ }^{29-32,44,59-61}$ but because individuals with SAD show residual symptoms of social anxiety after effective treatment, normalized neural response in isolation may not solely explain reduced symptom severity. This further underscores the importance of taking multimodal measures of brain function and structure into account. ${ }^{10}$

The interrelationship between neuronal response changes and the underlying anatomical plasticity is not clearly established in the animal literature. ${ }^{62,63}$ However, a large body of research exists on fear and anxiety in the brain, $8,64,65$ suggesting that both the number of recruited amygdala neurons ${ }^{14}$ and the strength of the neuronal response correlate with anxious behavior in rats. ${ }^{66}$ Trabalza et $a l^{2}$ showed structural rearrangements in the mice amygdala, the density of spines and number of nodes increasing after fear conditioning. Chronic stress also induces the formation of new synapses in the amygdala. ${ }^{67}$ Similarly, the total number and the size of synapses are reversed during fear extinction, ${ }^{68,69}$ that is, the laboratory analog to exposure interventions in CBT. ${ }^{70}$ Thus, we speculate that the attenuated anxiety-related amygdala volume could be due to synaptic reorganization, such as changes in spine shape or density, or a reduced number of synapses. However, in the present study the morphological neuroplasticity was related only to decreased state-dependent (anticipatory speech) anxiety, and not to more enduring symptoms of social anxiety as measured with the LSAS-SR.

Although the RCT design and multimodal-imaging approach are noteworthy strengths of the present study, there are also a number of limitations. As discussed elsewhere, ${ }^{27,42}$ the number of participants in the present study is limited, yet, sufficient to detect a differential treatment effect between CBT and ABM. The mediation analysis is also limited by not including a second repeated MRI assessment, that is, predictor and mediator were assessed at the same time-point. In addition, structural neuroplasticity and functional changes were closely related, but they may still be independently controlled by other processes such as metabolism, $, 1,72$ or the corticotropin-releasing factor system. ${ }^{3}$ Reduced amygdala neural response after CBT did not correlate with improved social anxiety. It is, however, likely that the taskspecific neural activations to self-referential criticism target cognitions not covered in our measures of state-dependent anxiety or LSAS-SR symptoms. Finally, eight SAD participants were on concurrent psychotropic medication, so we cannot entirely rule out drug $\times$ treatment interactions. However, these participants were evenly distributed in the trial arms.

In conclusion, we demonstrate compelling evidence that CBT for a common anxiety disorder simultaneously changes the physical structure and neurofunctional response of the amygdala. While our results support that amygdala neuroplasticity is directly related to improved social anxiety symptoms with CBT, these results should be replicated and further tested in other anxiety disorders and with other anxiolytic treatments.

\section{CONFLICT OF INTEREST}

The authors declare no conflict of interest.

\section{ACKNOWLEDGMENTS}

We are grateful to the staff of the Umeå Functional Brain Imaging Centre (UFBI) and Ann-Kathrine Larsson for providing excellent research conditions. Dr Malin GrenLandell conducted interviews with the healthy control group. Also, we thank the audience during behavioral tests: Amez Jaafar Hussein, Isabell Wincent, Johanna Henriksson, Karin Kamp, Kjell Andersson, Matts Östling, Morgan Ström, Sara Lann, Simon Nilsson and others. We also thank Katherine Cotter who provided us with proofreading. Parts of the data were presented in a poster at the 70th annual meeting of Society of Biological Psychiatry, 2015 in Toronto, Canada.

\section{DISCLAIMER}

Funding bodies had no role in the study setup, data interpretation or reporting. This study was supported by grants from Linköping University (GA), Swedish Research Council, Swedish Council for Working Life and Social Research (PC and TF), LJ Boëthius Foundation (TF) PRIMA Psychiatry Research Foundation (KNTM).

\section{REFERENCES}

1 Kühn S, Gleich T, Lorenz RC, Lindenberger U, Gallinat J. Playing Super Mario induces structural brain plasticity: gray matter changes resulting from training with a commercial video game. Mol Psychiatry 2014; 19: 265-271.

2 Trabalza A, Colazingari S, Sgobio C, Bevilacqua A. Contextual learning increases dendrite complexity and EphrinB2 levels in hippocampal mouse neurons. Behav Brain Res 2012; 227: 175-183.

3 Sztainberg Y, Kuperman Y, Tsoory M, Lebow M, Chen A. The anxiolytic effect of environmental enrichment is mediated via amygdalar CRF receptor type 1 . Mol Psychiatry 2010; 15: 905-917.

4 Lövdén M, Wenger E, Mårtensson J, Lindenberger U, Bäckman L. Structural brain plasticity in adult learning and development. Neurosci Biobehav Rev 2013; 37: 2296-2310.

5 Maass A, Duzel S, Goerke M, Becke A, Sobieray U, Neumann K et al. Vascular hippocampal plasticity after aerobic exercise in older adults. Mol Psychiatry 2015; 20: $585-593$.

6 Arnone D, McKie S, Elliott R, Juhasz G, Thomas EJ, Downey D et al. Statedependent changes in hippocampal grey matter in depression. Mol Psychiatry 2013; 18: 1265-1272.

7 Ghosh S, Laxmi TR, Chattarji S. Functional connectivity from the amygdala to the hippocampus grows stronger after stress. J Neurosci 2013; 33: 7234-7244.

8 Maren S, Quirk GJ. Neuronal signalling of fear memory. Nat Rev Neurosci 2004; 5: 844-852.

9 Poldrack RA. Imaging brain plasticity: conceptual and methodological issues-a theoretical review. Neurolmage 2000; 12: 1-13.

10 Sui J, Huster R, Yu Q, Segall JM, Calhoun VD. Function-structure associations of the brain: evidence from multimodal connectivity and covariance studies. NeuroImage 2014; 102: 11-23.

11 Nyberg L, Salami A, Andersson M, Eriksson J, Kalpouzos G, Kauppi K et al. Longitudinal evidence for diminished frontal cortex function in aging. Proc Natl Acad Sci USA 2010; 107: 22682-22686.

12 Salami A, Eriksson J, Nyberg L. Opposing effects of aging on large-scale brain systems for memory encoding and cognitive control. J Neurosci 2012; 32: 10749-10757. 
13 Redlich R, Grotegerd D, Opel N, Kaufmann C, Zwitserlood P, Kugel H et al. Are you gonna leave me? Separation anxiety is associated with increased amygdala responsiveness and volume. Soc Cogn Affect Neurosci 2015; 10: 278-284.

14 LeDoux J. The emotional brain, fear, and the amygdala. Cell Mol Neurobiol 2003; 23: 727-738.

15 Etkin A, Wager TD. Functional neuroimaging of anxiety: a meta-analysis of emotional processing in PTSD, social anxiety disorder, and specific phobia. Am J Psychiatry 2007; 164: 1476-1488.

16 Freitas-Ferrari MC, Hallak JEC, Trzesniak C, Filho AS, Machado-De-Sousa JP, Chagas $\mathrm{MHN}$ et al. Neuroimaging in social anxiety disorder: a systematic review of the literature. Prog Neuropsychopharmacol Biol Psychiatry 2010; 34: 565-580.

17 Shin LM, Liberzon I. The neurocircuitry of fear, stress, and anxiety disorders. Neuropsychopharmacol 2009; 35: 169-191.

18 Li L, Wu M, Liao Y, Ouyang L, Du M, Lei D et al. Grey matter reduction associated with posttraumatic stress disorder and traumatic stress. Neurosci Biobehav Rev 2014; 43: 163-172.

19 Talati A, Pantazatos SP, Schneier FR, Weissman MM, Hirsch J. Gray matter abnormalities in social anxiety disorder: primary, replication, and specificity studies. Biol Psychiatry 2013; 73: 75-84.

20 van Tol MJ, van der Wee NJ, van den Heuvel OA, Nielen MM, Demenescu LR, Aleman A et al. Regional brain volume in depression and anxiety disorders. Arch Gen Psychiatry 2010; 67: 1002-1011.

21 Radua J, van den Heuvel OA, Surguladze S, Mataix-Cols D. Meta-analytical comparison of voxel-based morphometry studies in obsessive-compulsive disorder vs other anxiety disorders. Arch Gen Psychiatry 2010; 67: 701-711.

22 Irle E, Ruhleder M, Lange C, Seidler-Brandler U, Salzer S, Dechent P et al. Reduced amygdalar and hippocampal size in adults with generalized social phobia. $J$ Psychiatry Neurosci 2010; 35: 126-131.

23 Frick A, Gingnell M, Marquand AF, Howner $K$, Fischer $H$, Kristiansson $M$ et al. Classifying social anxiety disorder using multivoxel pattern analyses of brain function and structure. Behav Brain Res 2014; 259: 330-335.

24 Frick $\mathrm{A}$, Howner $\mathrm{K}$, Fischer $\mathrm{H}$, Eskildsen SF, Kristiansson M, Furmark T. Cortical thickness alterations in social anxiety disorder. Neurosci Lett 2013; 536: 52-55.

25 Brühl AB, Delsignore A, Komossa K, Weidt S. Neuroimaging in social anxiety disorder-a meta-analytic review resulting in a new neurofunctional model. Neurosci Biobehav Rev 2014; 47: 260-280.

26 Liao W, Xu Q, Mantini D, Ding J, Machado-de-Sousa JP, Hallak JE et al. Altered gray matter morphometry and resting-state functional and structural connectivity in social anxiety disorder. Brain Res 2011; 1388: 167-177.

27 Månsson KNT, Carlbring P, Frick A, Engman J, Olsson C-J, Bodlund O et al. Altered neural correlates of affective processing after internet-delivered cognitive behavior therapy for social anxiety disorder. Psychiatry Res 2013; 214: 229-237.

28 Furmark T, Tillfors M, Marteinsdottir I, Fischer $\mathrm{H}$, Pissiota A, Langstrom B et al. Common changes in cerebral blood flow in patients with social phobia treated with citalopram or cognitive-behavioral therapy. Arch Gen Psychiatry 2002; 59: 425-433.

29 Lueken U, Straube B, Konrad C, Wittchen HU, Strohle A, Wittmann A et al. Neural substrates of treatment response to cognitive-behavioral therapy in panic disorder with agoraphobia. Am J Psychiatry 2013; 170: 1345-1355.

30 Goldin PR, Ziv M, Jazaieri H, Hahn K, Heimberg R, Gross JJ. Impact of cognitive behavioral therapy for social anxiety disorder on the neural dynamics of cognitive reappraisal of negative self-beliefs: randomized clinical trial. JAMA Psychiatry 2013; 70: 1048-1056.

31 Linden DEJ. How psychotherapy changes the brain - the contribution of functional neuroimaging. Mol Psychiatry 2006; 11: 528-538.

32 Phan KL, Coccaro EF, Angstadt M, Kreger KJ, Mayberg HS, Liberzon I et al. Corticolimbic brain reactivity to social signals of threat before and after sertraline treatment in generalized social phobia. Biol Psychiatry 2013; 73: 329-336.

33 Mahan AL, Ressler KJ. Fear conditioning, synaptic plasticity and the amygdala: implications for posttraumatic stress disorder. Trends Neurosci 2012; 35: 24-35.

34 Cassimjee N, Fouche J-P, Burnett M, Lochner C, Warwick J, Dupont P et al. Changes in regional brain volumes in social anxiety disorder following 12 weeks of treatment with escitalopram. Metab Brain Dis 2010; 25: 369-374.

35 Talati A, Pantazatos SP, Hirsch J, Schneier F. A pilot study of gray matter volume changes associated with paroxetine treatment and response in social anxiety disorder. Psychiatry Res 2015; 231: 279-285.

36 Schienle A, Wabnegger A, Scharmüller W. Effects of cognitive behavior therapy on regional brain volume in spider-phobic patients: preliminary results. J Anxiety Disord 2014; 28: 276-279.

37 Blair K, Geraci M, Devido J, McCaffrey D, Chen G, Vythilingam M et al. Neura response to self- and other referential praise and criticism in generalized social phobia. Arch Gen Psychiatry 2008; 65: 1176-1184.

38 First M, Gibbon M, Spitzer R, Williams J. Structured Clinical Interview for DSM-IV Axis I Disorders (SCID-I). American Psychiatric Press: Washington, DC, 1997.

39 Sheehan DV, Lecrubier Y, Sheehan KH, Amorim P, Janavs J, Weiller E et al. The Mini-International Neuropsychiatric Interview (M.I.N.I.): the development and validation of a structured diagnostic psychiatric interview for DSM-IV and ICD-10. J Clin Psychiatry 1998; 59: 22-33.

40 Baker SL, Heinrichs N, Kim HJ, Hofmann SG. The liebowitz social anxiety scale as a self-report instrument: a preliminary psychometric analysis. Behav Res Ther 2002 40: 701-715.

41 Svanborg P, Asberg M. A new self-rating scale for depression and anxiety states based on the comprehensive psychopathological rating scale. Acta Psychiatr Scand 1994; 89: 21-28.

42 Månsson KN, Frick A, Boraxbekk CJ, Marquand AF, Williams SC, Carlbring P et al. Predicting long-term outcome of Internet-delivered cognitive behavior therapy for social anxiety disorder using fMRI and support vector machine learning. Trans Psychiatry 2015; 5: e530.

43 Zaider TI, Heimberg RG, Fresco DM, Schneier FR, Liebowitz MR. Evaluation of the Clinical Global Impression Scale among individuals with social anxiety disorder. Psychol Med 2003; 33: 611-622.

44 Furmark T, Appel L, Michelgard A, Wahlstedt K, Ahs F, Zancan S et al. Cerebral blood flow changes after treatment of social phobia with the neurokinin-1 antagonist GR205171, citalopram, or placebo. Biol Psychiatry 2005; 58: 132-142.

45 Andersson G, Carlbring P, Furmark T. Group SOFIER Therapist experience and knowledge acquisition in internet-delivered CBT for social anxiety disorder: a randomized controlled trial. PLoS One 2012; 7: e37411.

46 Boettcher J, Carlbring P, Renneberg B, Berger T. Internet-based interventions for social anxiety disorder - an overview. Verhaltenstherapie 2013; 23: 160-168.

47 Andersson G, Cuijpers P, Carlbring P, Riper H, Hedman E. Guided Internet-based vs. face-to-face cognitive behavior therapy for psychiatric and somatic disorders: a systematic review and meta-analysis. World Psychiatry 2014; 13: 288-295.

48 MacLeod C, Mathews A. Cognitive bias modification approaches to anxiety. Annu Rev Clin Psychol 2012; 8: 189-217.

49 Carlbring P, Apelstrand M, Sehlin H, Amir N, Rousseau A, Hofmann SG et al. Internet-delivered attention bias modification training in individuals with social anxiety disorder--a double blind randomized controlled trial. BMC Psychiatry 2012 12: 66 .

50 Amir N, Beard C, Taylor CT, Klumpp H, Elias J, Burns M et al. Attention training in individuals with generalized social phobia: a randomized controlled trial. $J$ Consult Clin Psychol 2009; 77: 961-973.

51 Linetzky M, Pergamin-Hight L, Pine DS, Bar-Haim Y. Quantitative evaluation of the clinical efficacy of attention bias modification treatment for anxiety disorders. Depress Anxiety 2015; 32: 383-391.

52 Miskovic V, Schmidt LA. Social fearfulness in the human brain. Neurosci Biobehav Rev 2012; 36: 459-478.

53 Maldjian JA, Laurienti PJ, Kraft RA, Burdette JH. An automated method for neuroanatomic and cytoarchitectonic atlas-based interrogation of fMRI data sets. Neurolmage 2003; 19: 1233-1239.

54 Shrout PE, Bolger N. Mediation in experimental and nonexperimental studies: new procedures and recommendations. Psychol Methods 2002; 7: 422-445.

55 Casanova R, Srikanth R, Baer A, Laurienti PJ, Burdette JH, Hayasaka S et al. Biological parametric mapping: a statistical toolbox for multimodality brain image analysis. Neurolmage 2007; 34: 137-143.

56 Northoff G, Heinzel A, de Greck M, Bermpohl F, Dobrowolny H, Panksepp J. Selfreferential processing in our brain-A meta-analysis of imaging studies on the self. Neurolmage 2006; 31: 440-457.

57 Zhang W, Yang X, Lui S, Meng Y, Yao L, Xiao Y et al. Diagnostic prediction for social anxiety disorder via multivariate pattern analysis of the regional homogeneity. Biomed Res Int 2015; 2015: 763965.

58 Felmingham K, Kemp A, Williams L, Das P, Hughes G, Peduto A et al. Changes in anterior cingulate and amygdala after cognitive behavior therapy of posttraumatic stress disorder. Psychol Sci 2007; 18: 127-129.

59 Faria V, Ahs F, Appel L, Linnman C, Bani M, Bettica P et al. Amygdala-frontal couplings characterizing SSRI and placebo response in social anxiety disorder. Int J Neuropsychopharmacol 2014; 17: 1149-1157.

60 Faria V, Appel L, Ahs F, Linnman C, Pissiota A, Frans O et al. Amygdala subregions tied to SSRI and placebo response in patients with social anxiety disorder. Neuropsychopharmacology 2012; 37: 2222-2232.

61 Klumpp H, Fitzgerald DA, Phan KL. Neural predictors and mechanisms of cognitive behavioral therapy on threat processing in social anxiety disorder. Prog Neuropsychopharmacol Biol Psychiatry 2013; 45: 83-91.

62 Alvarez VA, Sabatini BL. Anatomical and physiological plasticity of dendritic spines. Annu Rev Neurosci 2007; 30: 79-97.

63 Nikonenko I, Jourdain P, Alberi S, Toni N, Muller D. Activity-induced changes of spine morphology. Hippocampus 2002; 12: 585-591.

64 Duvarci S, Pare D. Amygdala microcircuits controlling learned fear. Neuron 2014 82: $966-980$.

65 Wang YC, Ho UC, Ko MC, Liao CC, Lee L. Differential neuronal changes in medial prefrontal cortex, basolateral amygdala and nucleus accumbens after postweaning social isolation. Brain Struct Funct 2012; 217: 337-351. 
66 Ghosh S, Chattarji S. Neuronal encoding of the switch from specific to generalized fear. Nat Neurosci 2015; 18: 112-120.

67 Suvrathan A, Bennur S, Ghosh S, Tomar A, Anilkumar S, Chattarji S. Stress enhances fear by forming new synapses with greater capacity for long-term potentiation in the amygdala. Philos Trans R Soc Lond B Biol Sci 2014; 369: 20130151.

68 Heinrichs SC, Leite-Morris KA, Guy MD, Goldberg LR, Young AJ, Kaplan GB. Dendritic structural plasticity in the basolateral amygdala after fear conditioning and its extinction in mice. Behav Brain Res 2013; 248: 80-84.

69 Vetere G, Restivo L, Novembre G, Aceti M, Lumaca M, Ammassari-Teule M. Extinction partially reverts structural changes associated with remote fear memory. Learn Memory 2011; 18: 554-557.

70 Milad MR, Rosenbaum BL, Simon NM. Neuroscience of fear extinction: implications for assessment and treatment of fear-based and anxiety related disorders. Behav Res Ther 2014; 62: 17-23.
71 Mattsson N, Insel PS, Aisen PS, Jagust W, Mackin S, Weiner M et al. Brain structure and function as mediators of the effects of amyloid on memory. Neurology 2015; 84: $1136-1144$

72 Saxena S, Gorbis E, O'Neill J, Baker SK, Mandelkern MA, Maidment KM et al. Rapid effects of brief intensive cognitive-behavioral therapy on brain glucose metabolism in obsessive-compulsive disorder. Mol Psychiatry 2009; 14: 197-205.

\section{(c) (1)}

This work is licensed under a Creative Commons Attribution 4.0 International License. The images or other third party material in this article are included in the article's Creative Commons license, unless indicated otherwise in the credit line; if the material is not included under the Creative Commons license, users will need to obtain permission from the license holder to reproduce the material. To view a copy of this license, visit http://creativecommons.org/licenses/ by/4.0/

Supplementary Information accompanies the paper on the Translational Psychiatry website (http://www.nature.com/tp) 